spektiven Studie konnte gezeigt werden, dass eine Exzision des Lymphknotens schneller zum Erfolg führt als eine Antibiotikatherapie [Lindeboom JA et al. Clin Infect Dis 2007;44:1057-64]. Eine Antibiotikagabe allein bringt gegenüber einer abwartenden Haltung keine Vorteile [Lindeboom JA. Clin Infect Dis 2011;52:180-4]. Einer aktuellen Metaanalyse zufolge liegen die Heilungsraten bei kompletter Exzision bei $98 \%$, unter einer Antibiotikatherapie bei $73,1 \%$ und ohne Intervention bei $70,4 \%$ [Zimmermann $\mathrm{P}$ et al. J Infect
2015;71:9-18]. „Eine NTM-Lymphadenitis heilt bei immunkompetenten Kindern in der Regel auch ohne Behandlung spontan aus“, sagte Henneke. Die Heilungsdauer sei jedoch deutlich länger als bei einer Exzision. Allerdings kommt es nach einer Exzision bei $10 \%$ zu einer Fazialisparese. Die Vor- und Nachteile der verschiedenen Optionen sollten mit den Eltern ausführlich besprochen werden. Wenn operiert wird, sollte dies frühzeitig und sorgfältig, aber nicht radikal erfolgen. Antibiotika sollten nicht primär gegeben werden, eventuell aber bei inkompletter Operation oder ausgedehntem Befund. Bei Befall mehrerer Organe und Immundefizienz spielen Antibiotika jedoch eine wichtige Rolle. Sinnvoll ist allerdings nur eine Gabe über mindestens sechs Monate, betonte Henneke. Bei Infektionen mit M. avium oder M. intracellulare kommen Rifampicin plus Clarithromycin oder Azithromycin infrage, initial kann Ethambutol erwogen werden.

Angelika Bauer-Delto

Henneke P: NTM-Infektion - wann behandeln?

\section{CMV-Infektion bei Neugeborenen - eine Herausforderung}

Ein ine Infektion mit dem humanen Zytomegalievirus (CMV) ist die häufigste konnatale Infektion in Deutschland. Bei einer Geburtenrate von rund 700.000 Kindern pro Jahr ist bei einer geschätzten Inzidenz zwischen 0,2 und $0,5 \%$ von 1.400 bis $3.500 \mathrm{CMV}$-infizierten Neugeborenen auszugehen, berichtete Dr. Horst Buxmann, Klinik für Kinder- und Jugendmedizin, Universitätsklinikum Frankfurt. Für CMV-Infektionen ist jedoch weder im Rahmen der Schwangerenvorsorge noch bei Neugeborenen ein Screening etabliert.

Eine CMV-Infektion in der Schwangerschaft führt in rund $40 \%$ der Fälle zu einer konnatalen Infektion. Circa $10 \%$ der infizierten Kinder zeigen bei der Geburt Symptome, etwa jedes zehnte von ihnen verstirbt. Auch von den zunächst

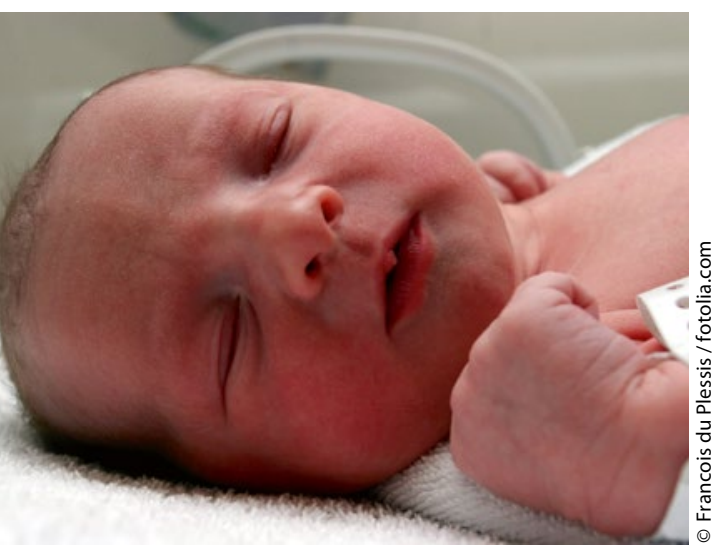

asymptomatischen Kindern entwickeln 10-15\% im weiteren Verlauf Beeinträchtigungen, vorwiegend des Hörvermögens, aber auch geistige Retardierungen sind beschrieben.

Häufige klinische Zeichen einer konnatalen CMV-Infektion sind thalamostriatale Vaskulopathien, Petechien, Ikterus, Mikrozephalie, Wachstumsretardierung, Hörstörungen bis zur Taubheit, Chorioretinitis, Migrationsstörungen des ZNS, zerebrale Krampfanfälle, Pneumonie und Enteritis.

Bei anamnestischen Hinweisen auf eine CMV-Infektion der Mutter während der Schwangerschaft oder bei Symptomen des Neugeborenen, die auf eine konnatale CMV-Infektion hinweisen, ist innerhalb der ersten zwei Lebenswochen eine Labordiagnose durchzuführen [AWMF-Leitlinie Register Nr. 093-001]. Geeignet sind hierfür der Nachweis von CMV-DNA und die Isolierung von CMV aus Urin oder von der Mundschleimhaut des Neugeborenen mittels Virusabstrich.

Insbesondere bei einer CMV-Infektion mit Symptomen des zentralen Nervensystems wird eine antivirale Therapie mit intravenösem Ganciclovir oder oralem Valganciclovir empfohlen. Diese Medikamente sind für Neugeborene allerdings nicht zugelassen. Wie aktuelle Daten zeigen, kommt es unter der Therapie zu einer relevanten Besserung des Hörvermögens [Bilavsky E et al. Arch
Dis Child 2016;101:433-8]. Die neurologische Entwicklung bleibe jedoch trotz aufwendiger antiviraler Therapie bei vielen Kindern beeinträchtigt, so Buxmann.

Zudem kann eine Vielzahl unerwünschter Wirkungen auftreten, insbesondere reversible Neutropenien und Thrombozytopenien. Die Eltern sind darüber sorgfältig aufzuklären, betonte Buxmann. $\mathrm{Zu}$ möglichen Langzeitnebenwirkungen der Behandlung liegen bislang keine Daten vor. Für asymptomatische Neugeborene mit CMVInfektion wird derzeit keine antivirale Therapie empfohlen, auch wenn es im weiteren Verlauf zu bleibenden Schäden bei den betroffenen Kindern kommen kann.

„Insgesamt ist die therapeutische Situation unbefriedigend“, resümierte Buxmann. Umso wichtiger sei daher die Prävention. Schwangere und Frauen, die eine Schwangerschaft planen, sollten den direkten Kontakt zu Körperflüssigkeiten von Kleinkindern und Säuglingen möglichst vermeiden, also die Kinder nicht auf den Mund küssen sowie Geschirr oder Zahnbürsten nicht gemeinsam benutzen und regelmäßig, insbesondere nach dem Windelwechseln und nach Kontakt mit Nasensekret, Speichel, Tränen oder Urin die Hände waschen. Hygienemaßnahmen können die CMV-Serokonversionsrate in der Schwangerschaft und die Inzidenz von konnatalen CMV-Infektionen deutlich senken.

Angelika Bauer-Delto

Buxmann H: Zytomegalie Virus. 